\title{
Boost Irradiation Integrated to Whole Brain Radiotherapy in the Management of Brain Metastases
}

\author{
Ágnes Dobi ${ }^{1} \cdot$ Emese Fodor ${ }^{1}$ - Anikó Maráz ${ }^{1} \cdot$ Zsófia Együd $^{1} \cdot$ Adrienne Cserháti $^{1} \cdot$ László Tiszlavicz $^{2} \cdot$ Zita Reisz $^{2}$. \\ Pál Barzó $^{3} \cdot$ Zoltán Varga $^{1} \cdot$ Katalin Hideghéty ${ }^{1}$
}

Received: 4 June 2017 / Accepted: 10 January 2018

(C) Arányi Lajos Foundation 2018

\begin{abstract}
Our retrospective analysis aimed to evaluate the clinical value of dose intensification schemes: WBRT and consecutive, delayed, or simultaneous integrated boost (SIB) in brain metastasis (BM) management. Clinical data and overall survival (OS) of 468 patients with BM from various primaries treated with $10 \times 3$ Gy WBRT $(n=195)$, WBRT $+10 \times 2$ Gy boost $(n=125)$, or simultaneously $15 \times 2.2$ Gy WBRT +0.7 Gy boost $(n=148)$ during a 6-year period were statistically analysed. Significant difference in OS could be detected with additional boost to WBRT (3.3 versus 6.5 months) and this difference was confirmed for BMs of lung cancer and melanoma and both for oligo- and multiplex lesions. The OS was prolonged for the RPA 2 and RPA3 categories, if patients received escalated dose, 4.0 vs. 7.7 months; $(p=0.002)$ in class RPA2 and 2.6 vs. 4.2 months; $(p<0.0001)$ in the class RPA 3 respectively. The significant difference in OS was also achieved with SIB. The shortened overall treatment time of SIB with lower WBRT fraction dose exhibited survival benefit over WBRT alone, and could be applied for patients developing BM even with unfavourable prognostic factors. These results warrant for further study of this approach with dose escalation using the lately available solutions for hippocampus sparing and fractionated stereotactic irradiation. The simultaneous delivery of WBRT with reduced fraction dose and boost proved to be advantageous prolonging the OS with shortened treatment time and reduced probability for cognitive decline development even for patients with poor performance status and progressing extracranial disease.
\end{abstract}

Keywords WBRT $\cdot$ Simultaneous integrated boost $\cdot$ Brain metastasis $\cdot 3$ DCRT $\cdot$ IMRT

\section{Introduction}

Alongside the improved efficacy of systemic treatments, the incidence of brain metastases (BMs) steadily increases, ranging 9-40\% worldwide [1,2]. The most common cancers that metastasise to the brain include lung, breast, melanoma, renal cell, and colorectal cancers [3]. The recent advances in chemotherapy and the fast growing introduction of diverse molecular targeted approaches (including EGFR-, VEGFR-,

Ágnes Dobi

dobiagnes@gmail.com

1 Department of Oncotherapy, University of Szeged, Korányi fasor 12, Szeged H-6720, Hungary

2 Department of Pathology, University of Szeged, Állomás utca 1, Szeged H-6725, Hungary

3 Department of Neurosurgery, University of Szeged, Semmelweis utca 8, Szeged H-6725, Hungary multikinase, B-RAF, MEK, ERK and immunoresponse CTLA-4, PD-1, PD-L1 inhibitors) into the management of these metastatic cancers lead to remarkable improvement in the outcome of disseminated stage of these frequent tumours. In the case of brain metastasis, though, the overall prognosis remains poor: without any treatment $1-2$ months, with palliative methods, 4-6 months of median survival can be expected [4], depending (1) on the age and functional status of the patient, (2) the extent of the underlying systemic disease, and (3) the number of metastases [5]. Moreover, in 15\% of the cases, the primary tumour is still unknown at the time when BMs are already diagnosed [4].

The common therapeutic mean is the palliative irradiation with different possible methods to choose from. In case of multiple BMs, the whole-brain irradiation (WBRT) is usually performed with the dose of $10 \times 3$ Gy [6], whilst the cases with less than three metastatic lesions (i.e. oligometastases) were considered for surgery and/or radiosurgery with or without WBRT [7, 8]. In the last decade, conclusions from relevant clinical studies demonstrated the superiority of multimodal 
approaches over the single treatment method of neurosurgery, stereotactic radiosurgery (SRS), and WBRT [9-11]. More recently, in order to decrease the cognitive decline attributed to the standard WBRT, the possibility of omitting the WBRT came again into focus. The ASCO recommendation in 2014 did not include the routine addition of WBRT to SRS for limited brain metastases, based on meta-analysis of published clinical research $[12,13]$. However, it could be substantially criticised and several arguments (including survival benefit, notable increase of intracerebral impairments, and more serious neurocognitive decline due to metastatic progression in the brain) support the further application of combined approaches including the WBRT $[14,15]$.

At the same time, any mean to mitigate the treatmentrelated brain toxicities, such as the use of radioprotective agents, and/or hippocampus-sparing advanced radiation technique and/or alternative fractionation of WBRT, is highly required. The conformational fractionated external beam boost, instead of SRS for BMs arising from lung cancer (as it has been recently demonstrated in a multi-institutional study [16]), results in a remarkable estimated 1-year local control rate of $>75 \%$. Such dose escalation can be also carried out with intensity-modulated radiotherapy (IMRT) or volumetric modulated arc therapy (VMAT), using the simultaneous integrated boost (SIB) technique $[8,17]$, or, if the above mentioned techniques are not available, with external beam radiotherapy boost (RTB) [16].

Several technical and logistical advantages of SIB over other modalities (such as radiosurgery) makes SIB a plausible choice. SIB includes WBRT and boost on BM in the same session with optimised dose distribution, requires a single simulation protocol, reducing the waiting and dose delivery time, therefore costs and inconvenience, as well [18]. Using single fraction protocols, the re-oxygenation and re-assortment benefits accompanying fractionated modalities, cannot be exploited. The treatment of more than 3 metastases with radiosurgery may require long or multiple sessions, or may lead to the impossibility of radiosurgery, as well. The substantial time break between radiosurgery and WBRT may enhance the probability of tumour cell repopulation or subletal repair processes [19].

From 2005 on, the dose escalation with 3D conformal technique was introduced for patients presented with brain metastasis at our Department, in the lack of SRS availability before 2016. The standard WBRT and the new approaches were performed parallel; therefore we could evaluate the results of the different radiation schemes in a single institutional retrospective analysis.

\section{Materials and Methods}

Between 2005 and 2013, at the Department of Oncotherapy, altogether 468 patients with BMs (arising from various primary malignancies) were subjected to palliative skull irradiation. The present study has been carried out in accordance with The Code of Ethics of the World Medical Association (Declaration of Helsinki) for experiments involving humans. Informed consent was obtained from the patients at their first clinical admission for the anonymised use of their patient data for research purposes. For the present study, the ethical permission (No. 886/2006) was issued by the Ethical Council of the Faculty of Medicine, University of Szeged. The treatment schemes were thoroughly discussed with every single patient, independently from their actual performance status. The fractionation was agreed by signed informed consent. Unconscious patients or those with serious cognitive deficits, lacking the legal capacity of informed consent made on their own, and also patients with previous prophylactic WBRT, were excluded from the enrolment. All patients included in the present study were treated according to the actual protocol for the histologically proven primary disease and had clinical, radiological, pathological evidence of BM.

The total biological effective dose was calculated as equivalent dose in 2 Gy fractions (EQD2: $\alpha / \beta$ value of 2 for normal brain [Gy2] and that of 10 for tumour [Gy10]). The traditional palliative approach of $10 \times 3$ Gy WBRT (EQD2 37.5 Gy) was applied for 195 cases (Group A); in 273 cases WBRT combined with boost irradiation were performed. In addition to the $10 \times 3$ Gy $/ 18 \times 2$ Gy WBRT boost dose of $10 \times 2$ Gy $($ EQD2 $57,5 \mathrm{~Gy} / 56 \mathrm{~Gy}$ ) to the surgical cavity (if the metastasis had been removed), or to the metastatic lesions for patients in good PS and/or better life expectancy were performed (Group B). Later, simultaneous integrated boost irradiation (SIB: $15 \times 2.2$ WBRT $+15 \times 0.7$ boost, (WBRT EQD2 33,4Gy, metastasis EQD2 46.8 Gy) (Group C) had been given whenever it was applicable with 3D conformal technique planned by XIO TPS.

Patients were immobilised with a 3-point thermoplastic mask (ORFIT Industries, NL). Radiotherapy was planned according to the ICRU 52 recommendation using subfields additionally to the opposed lateral fields to achieve the required dose homogeneity (95-107\%) for WBRT. Boost using 3 DCRT or IMRT at $10 \times 2$ Gy boost was given consecutively or with 2-3 months delay, due to the treatment of either the primary tumour or of the extracranial metastases. Later, we have developed the technique of conformal simultaneous boost, delivered whenever the metastases could be encompassed in a boost volume less than $30 \%$ of the brain (in majority of the cases less than $10 \%$ ). Boost volume was defined on the basis of planning CAT and MRI fusion. During brain irradiation patients received $12 \mathrm{mg}$ methylprednisolone for prevention of brain oedema, with gradually decreased dosing after radiotherapy. The dose of methylprednisolone was adjusted according to the symptoms of intracranial pressure elevation due to brain oedema. Retrospective assessment of overall survival (OS) according to the recursive partitioning analysis (RPA), Karnofsky performance score 
(KPS), number of metastases, metastasectomy, localisation and histological features of primary tumour was carried out. The data were evaluated by Kaplan-Meier statistical analysis with IBM SPSS Statistics for Windows, Version 20.0 (Armonk, NY: IBM Corp.) $p$ value $<0.05$ was considered as statistically significant. Multi-variance analysis of the prognostic factors was performed using the Cox proportional hazard regression model.

\section{Results}

\section{Patient Characteristics}

The mean age of the population at the time of BM detection was 60.7 years; $53.3 \%$ of them was male and $46.7 \%$ female. The KPS was in $56.2 \%$ of this population over $70 \%$. The primary neoplasm was that of the lung in $68.9 \%$ (16\% of the latter was small cell lung cancer without prior prophylactic skull irradiation), of the breast in $7.9 \%$, malignant melanoma in $10.6 \%$, of the kidney in $2.1 \%$, of the gastrointestinal tract in $5.4 \%$, or that of other organ in $4.9 \%$ of the assessed population. $36 \%$ of patients with small cell lung cancer received dose-escalated radiotherapy, the remaining cases were treated with WBRT. At the time of the diagnosis of the cerebral metastasis, the underlying process was controlled in $37.4 \%$, extracranial progression in $35.8 \%$ and was unknown in $26.6 \%$ of this population. $11.1 \%$ of all patients belonged to RPA 1 , $45.1 \%$ to RPA 2, and $43.8 \%$ to RPA 3 class. During the treatments, the data of the patients were registered according to the RPA categories; therefore the graded prognostic assessment (GPA) could not be defined retrospectively in lack of Karnofsky index between 70 and 100 . Nevertheless, the treatment outcome could be evaluated according to the number of brain metastases.

Table 1 summarises the patient characteristics according to the three different methods of treatment. Patient distribution according to the age, gender and primary tumour localisation, extracranial metastasis were similar in the 3 groups. The difference of the KPS at the diagnosis and consequently the patient distribution according to the RPA categories shows the patient selection strategy at our Department. In RPA 2, the primary tumour was controlled in $13 \%$, uncontrolled in $63 \%$, unknown in $24 \%$ of the cases. In RPA 3, this distribution was $10 \%, 78 \%$, and $12 \%$, respectively.

\section{Characteristics of the Malignant Disease and Brain Metastases}

Extracranial metastasis was present $53 \%$ in the RPA 2, and $56 \%$ of the cases in the RPA 3 group. Extracranial metastasis was not present in $30 \%$ and $38 \%$, or was unknown in $17 \%$ and $6 \%$ of the cases in RPA 2 and 3 classes, respectively. Higher number of the patients received high dose radiotherapy with good PS, with better prognostic factors and with solitary or oligometastases. The difference according to the KPS and RPA was less pronounced in the Group C, then in the Group B over the Group A. Even the status of extracranial disease was less favourable in the Group $\mathbf{C}$ with $50 \%$ progressive disease, in contrast to $36 \%$ in the Group A and $21 \%$ in the Group B. Table 2 characterises the brain metastases further. The time frame of brain metastasis occurrence had a relation to the underlying disease, hence were similarly distributed between the treatment groups. The development of brain metastases occurred within 1 year after the diagnosis of primary tumour in $72 \%$ of the patients in Group A, $66 \%$ in Group B and $64 \%$ in Group C. Late occurring brain metastases were diagnosed in $13.8 \%, 14.4 \%$ and $17.6 \%$ in the Groups A, B, C, respectively. The rate of single-/oligometastases was higher than two third of the group when the higher total dose was applied (Groups B and C), and was $42 \%$ of the patients received WBRT only. Consequently, only $10 \%$ of the patients of Group A underwent surgery, meanwhile almost the half of the patients was subjected to neurosurgical removal of the metastases in the other two groups. 147 patients were subjected to brain metastasectomy, $90 \%$ of them also received postoperative/ adjuvant radiotherapy, independently from the radiotherapy modality. The planning target volume (PTV) for the WBRT was similar in the three groups. The difference between the boost PTVs of the Groups B (153.3 $\left.\pm 27.9 \mathrm{~cm}^{3}\right)$ and $\mathrm{C}\left(183.1 \pm 15.7 \mathrm{~cm}^{3}\right)$ was statistically significant $(p=0.017)$.

\section{Survival Analysis}

The analysis of OS according to different factors is summarised in Table 3. Doubling of the survival time was detected in the escalated dose groups over the Group A. $(p<0.001)$ The OS was 3.2-3.3 months for all tumour types, if only WBRT was applied. OS difference was significant in the case of lung cancer and malignant melanoma between patients treated by WBRT only vs. those receiving escalated total dose. OS difference has not reached the statistical significance level for breast, kidney and gastrointestinal tumours. Both in the case of low number of the BM (1-3) and in the case of multiple $(>4)$ metastases, the OS difference between the $30 \mathrm{~Gy}$ and escalated groups were significant. If surgery was performed, statistically no OS benefit could be proven from the boost dose $(p=0.48)$, in contrast to the significantly prolonged survival without neurosurgical removal $(p=$ 0.002). The longer treatment with higher total dose (SIB or consecutive boost to WBRT) was significantly more beneficial for the survival of patients both in good and in poor condition (Table 4). The survival data of the Group B and C in RPA classes 2 and 3 were significantly better than in Group A. Only a few patients in RPA class 1 were treated with WBRT, therefore in spite of the median survival of Group B 
Table 1 Patient characteristics by treatment groups

\begin{tabular}{|c|c|c|c|c|}
\hline Treatment group & $\begin{array}{l}\text { A WBRT } \\
10 \times 3 G y \\
n=195\end{array}$ & $\begin{array}{l}\text { B WBRT }+ \text { boost } \\
10 \times 3 / 18 \times 2+10 \times 2 \\
n=125\end{array}$ & $\begin{array}{l}\text { C SIB } \\
15 \times(2.2+0.7) \\
n=148\end{array}$ & $\begin{array}{l}\mathrm{B}+\mathrm{C} \text { group } \\
n=273\end{array}$ \\
\hline \multicolumn{5}{|l|}{ Age } \\
\hline Mean & 60.9 & 58.3 & 63.1 & 60.7 \\
\hline Range & $27.6-84.2$ & $21.6-84.7$ & $38.3-84.8$ & $21.6-84.8$ \\
\hline$<50$ & $20(10.3 \%)$ & $28(22.4 \%)$ & $10(6.8 \%)$ & $38(13 \%)$ \\
\hline $50-70$ & $135(69.2 \%)$ & $78(62.4 \%)$ & $103(69.6 \%)$ & $181(66 \%)$ \\
\hline$>70$ & $40(20.5 \%)$ & $19(15.2 \%)$ & $35(23.6 \%)$ & $54(19 \%)$ \\
\hline \multicolumn{5}{|l|}{ Gender } \\
\hline Male & $107(54.9 \%)$ & $69(55.2 \%)$ & $74(50 \%)$ & $143(52 \%)$ \\
\hline Female & $88(45.1 \%)$ & $56(44.8 \%)$ & $74(50 \%)$ & $130(48 \%)$ \\
\hline \multicolumn{5}{|l|}{ Primary tumour } \\
\hline Lung (total) & $143(73.3 \%)$ & $85(68 \%)$ & $97(65.6 \%)$ & $182(66 \%)$ \\
\hline$-S C L C$ & $35(17.9 \%)$ & $7(5.6 \%)$ & $13(8.7 \%)$ & $20(7.3 \%)$ \\
\hline$-N S C L C$ & $108(55.3 \%)$ & $78(62.4 \%)$ & $84(56.7 \%)$ & $162(59.3 \%)$ \\
\hline Breast & $16(8.2 \%)$ & $7(5.6 \%)$ & $15(10.1 \%)$ & $22(8 \%)$ \\
\hline $\mathrm{MM}$ & $9(4.6 \%)$ & $18(14.4 \%)$ & $19(12.8 \%)$ & $37(13 \%)$ \\
\hline Kidney & $5(2.6 \%)$ & $3(2.4 \%)$ & $2(1.4 \%)$ & $5(1.8 \%)$ \\
\hline Colorectal & $10(5.1 \%)$ & $8(6.4 \%)$ & $7(4.7 \%)$ & $15(5.4 \%)$ \\
\hline Other & $12(6.2 \%)$ & $4(3.2 \%)$ & $8(5.4 \%)$ & $12(4.3 \%)$ \\
\hline \multicolumn{5}{|l|}{ KPS } \\
\hline$>70$ & $81(41.5 \%)$ & $93(74.4 \%)$ & $78(52.7 \%)$ & $171(62 \%)$ \\
\hline$<70$ & $114(58.5 \%)$ & $32(25.6 \%)$ & $70(47.3 \%)$ & $102(37 \%)$ \\
\hline \multicolumn{5}{|c|}{ Status of extracranial dissemination } \\
\hline No. & $67(34.4 \%)$ & $63(50.4 \%)$ & $19(12.8 \%)$ & $82(30 \%)$ \\
\hline Regression & $10(5.1 \%)$ & $8(6.4 \%)$ & $5(3.4 \%)$ & $13(4.7 \%)$ \\
\hline Progression & $70(35.9 \%)$ & $27(21.6 \%)$ & $74(50 \%)$ & $101(37 \%)$ \\
\hline Unknown & $48(24.6 \%)$ & $27(21.6 \%)$ & $50(33.8 \%)$ & $77(28 \%)$ \\
\hline \multicolumn{5}{|l|}{ RPA } \\
\hline 1 & $8(4.1 \%)$ & $28(22.4 \%)$ & $10(6.8 \%)$ & $38(14 \%)$ \\
\hline 2 & $73(37.4 \%)$ & $65(52 \%)$ & $68(45.9 \%)$ & $133(48 \%)$ \\
\hline 3 & $114(58.5 \%)$ & $32(25.6 \%)$ & $70(47.3 \%)$ & $102(38 \%)$ \\
\hline
\end{tabular}

$\mathrm{MM}=$ malignant melanoma

(20.2 months) and in Group C (14.6 months) in contrast to the 6.3 months of Group A; it has not reached the statistical significance (Table 4). Kaplan-Meier survival analysis detected no significant OS difference between the treatment groups for the patients of RPA 1 category. As for the RPA 2 and RPA3 categories, the OS was significantly prolonged in case of patients received escalated dose, 4.0 vs. 7.7 months; $(p=0.002)$ in class RPA2 and 2.6 vs. 4.2 months; $(p<0.0001)$ in the class RPA 3. If no surgery was performed the SIB resulted in significantly longer OS of 6.5 months in contrast to the 3.9 months survival of the patients received WBRT only for class $1-2(p=0.05)$. In RPA class 3 , the addition of both the consecutive or delayed boost and the simultaneous boost to the WBRT resulted in significant OS benefit $(p=0.001)$. The
OS of patients with KPS $>70 \%$ and even the OS of patients with KPS $<70 \%$ were equally proven better in case of those receiving the escalated dose vs. WBRT without boost (9.4 vs. 4.2 months; $p<0.0001$ and 4.2 vs. 2.6 months; $\mathrm{p}<0.0001$; respectively). The multi-variance analysis yielded three, mutually independent prognostic factors for survival: RPA, surgery and therapy method. According to this analysis, the number of metastases, as a prognostic factor, is not independent from the ones mentioned above (as shown in Table 5).

\section{Toxicity of the Treatment}

In the majority of the cases in each group, the prophylactic dose of the methyl-prednisolone prevented the development 
Table 2 Characteristics of brain metastases by treatment groups

\begin{tabular}{|c|c|c|c|}
\hline Treatment group & $\begin{array}{l}\text { A WBRT } \\
10 \times 3 \mathrm{~Gy}\end{array}$ & $\begin{array}{l}\text { B WBRT }+ \text { boost } \\
10 \times 3 / 18 \times 2+10 \mathrm{X} 2\end{array}$ & $\begin{array}{l}\text { C SIB } \\
15 \times(2.2+0.7)\end{array}$ \\
\hline \multicolumn{4}{|l|}{ Development of BM after the diagnosis of the primary } \\
\hline First manifestation of the disease or within 1 year & $140(71.8 \%)$ & $83(66.4 \%)$ & $95(64.2 \%)$ \\
\hline $1-3$ years & $28(14.4 \%)$ & $24(19.2 \%)$ & $27(18.2 \%)$ \\
\hline$>4$ years & $27(13.8 \%)$ & $18(14.4 \%)$ & $26(17.6 \%)$ \\
\hline \multicolumn{4}{|l|}{ Number of BM } \\
\hline average & 5,1 & 2,2 & 2,9 \\
\hline 1 & $38(19.5 \%)$ & $78(62.4 \%)$ & $69(46.6 \%)$ \\
\hline $2-4$ & $44(22.6 \%)$ & $29(23.2 \%)$ & $50(33.8 \%)$ \\
\hline$>4$ & $113(57.9 \%)$ & $18(14.4 \%)$ & $29(19.6)$ \\
\hline \multicolumn{4}{|l|}{ BM surgery } \\
\hline no & $176(90.3 \%)$ & $63(50.4 \%)$ & $82(55.4 \%)$ \\
\hline yes & $19(9.7 \%)$ & $62(49.6 \%)$ & $66(44.6 \%)$ \\
\hline \multicolumn{4}{|l|}{ RT volumes of interest } \\
\hline PTV & 1705 & 1716 & 1686 \\
\hline PTV1 & & 153.3 & 183.1 \\
\hline Brain & 1326 & 1341 & 1314 \\
\hline GTV & & 35.9 & 59.1 \\
\hline
\end{tabular}

of serious brain oedema, the consequent intracranial pressure elevation, and the aggravation of the neurological symptoms. In $35 \%$ of the cases, the dehydration had to be intensified, but with appropriate supportive control, $96 \%$ of the patients could complete the planned therapy. Alopecia occurred in all groups equally.

\section{Discussion}

There is still a seething debate concerning the optimal management of brain metastases. The main aim that everyone agrees with is to achieve as long time for the patients as possible without physical and psychological signs and symptoms.

Table 3 The OS directly related to the type of irradiation, primary tumour, number of brain metastases and metastasectomy

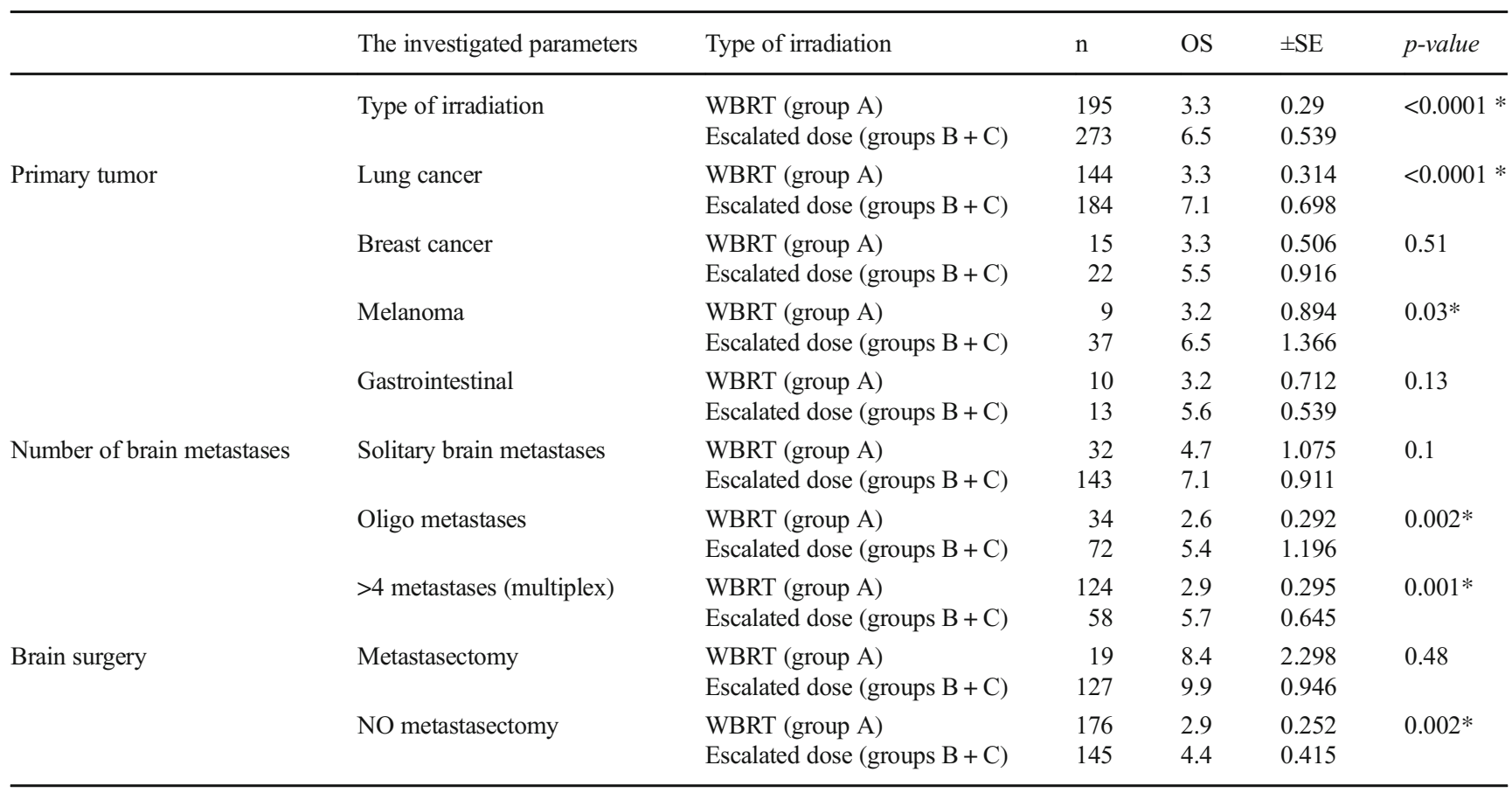


Table 4 The OS as a function of Karnovsky-scores or RPA

\begin{tabular}{|c|c|c|c|c|c|}
\hline The investigated parameters & Type of irradiation & $\mathrm{n}$ & OS & $\pm \mathrm{SE}$ & p-value \\
\hline \multirow[t]{4}{*}{ Karnofsky > 70\% } & \multirow[t]{2}{*}{ WBRTSIB } & 81 & 4.1 & 0.45 & \multirow[t]{2}{*}{0.015} \\
\hline & & 78 & 8.9 & 0.867 & \\
\hline & \multirow[t]{2}{*}{ WBRTWBRT+boost } & 81 & 4.1 & 0.45 & \multirow[t]{2}{*}{$<0.0001$} \\
\hline & & 93 & 9.6 & 0.868 & \\
\hline \multirow[t]{4}{*}{ Karnofsky $<70 \%$} & \multirow[t]{2}{*}{ WBRTSIB } & 114 & 2.6 & 0.19 & \multirow[t]{2}{*}{0.002} \\
\hline & & 70 & 3.9 & 0.465 & \\
\hline & \multirow[t]{2}{*}{ WBRTWBRT+boost } & 114 & 2.6 & 0.19 & \multirow[t]{2}{*}{0.021} \\
\hline & & 32 & 4.2 & 0.636 & \\
\hline \multirow[t]{4}{*}{ RPA1 } & \multirow[t]{2}{*}{ WBRTSIB } & 8 & 6.3 & 6.223 & \multirow[t]{2}{*}{0.343} \\
\hline & & 10 & 20.2 & 2.972 & \\
\hline & \multirow[t]{2}{*}{ WBRTWBRT+boost } & 8 & 6.3 & 6.223 & \multirow[t]{2}{*}{0.345} \\
\hline & & 28 & 14.6 & 6.456 & \\
\hline \multirow[t]{4}{*}{ RPA2 } & \multirow[t]{2}{*}{ WBRTSIB } & 73 & 4.0 & 0.427 & \multirow[t]{2}{*}{0.034} \\
\hline & & 68 & 7.7 & 1.052 & \\
\hline & \multirow[t]{2}{*}{ WBRTWBRT+boost } & 73 & 4.0 & 0.427 & \multirow[t]{2}{*}{0.003} \\
\hline & & 65 & 8.3 & 1.875 & \\
\hline \multirow[t]{4}{*}{ RPA3 } & \multirow[t]{2}{*}{ WBRTSIB } & 114 & 2.6 & 0.19 & \multirow[t]{2}{*}{0.002} \\
\hline & & 70 & 3.9 & 0.465 & \\
\hline & \multirow[t]{2}{*}{ WBRTWBRT+boost } & 114 & 2.6 & 0.19 & \multirow[t]{2}{*}{0.021} \\
\hline & & 32 & 4.2 & 0.636 & \\
\hline
\end{tabular}

Historically, the very short survival without treatment, with the symptoms of increasing intracranial pressure and diverse neurological deteriorations including motor-, sensory-, speech- and cognitive dysfunctions led to delivery of palliative 30 Gy WBRT, in 10 fractions [20]. Ten or five consecutive working days were reasonable even for patients in poor condition with short life expectancy and the RT was performed with simple technique (two opposed lateral beams) with relative large dose inhomogeneity in the whole brain. Nevertheless, WBRT resulted in symptom relief in the majority of the patients, and added some months to their survival [21]. Based on that, WBRT became the standard management of brain metastasis [22] and had been also introduced as preventive measure in case of radiosensitive malignancy with high risk for BM or leptomeningeal dissemination (ALL, SCLC) $[23,24]$. The prophylactic WBRT is performed with conventional fractionation (1.8-2 Gy/fraction). There is no doubt that WBRT is highly effective to prevent metastatic spread in the brain, but severe neurocognitive decline

Table 5 RPA, surgery and RT method, as prognostic factors for survival

\begin{tabular}{lllll}
\hline Risk factors & HR & $95 \%$ CI & $p$-value \\
\hline RPA & & & & $<0.001$ \\
& RPA 3 vs. RPA 1 & 3.546 & $2.463-5.102$ & \\
& RPA 3 vs. RPA 2 & 1.773 & $1.441-2.183$ & \\
Surgery & NO vs. YES & 2.072 & $1.643-2.512$ & $<0.001$ \\
RT group & Group A vs. Group B,C & 1.256 & $1.021-1.546$ & 0.031 \\
\hline
\end{tabular}

(particularly memory deficit) occurs with high probability after $5 \times 4$ Gy or $10 \times 3$ Gy [25]. The introduction of stereotactic radiosurgery technique in the 1960 s provided access to highly selective dose delivery method for small $(<3 \mathrm{~cm})$, and low number (1-3) of brain metastases, which was applied as alternative method to neurosurgical intervention. Several clinical studies proved the superiority of the combined treatment, surgery or SRS + WBRT to improve local and central nervous system control [10, 26-29]. In the last decade, with the advent of imaging (CAT/MRI), development of neurosurgery technique and various SRS solutions, high precision local management of single and oligo-BM became widely available. Recent clinical trials and meta-analyses questioned the necessity of WBRT at all, emphasising the neurocognitive harm of the WBRT [12, 30-34]. Meanwhile, other authors [35] warn of drawing too early and hasty conclusions leading to general omission of WBRT, without considering the danger of increase of intracranial impairments, and neurocognitive decline due to cerebral disease progression. They rather suggest considering the use of selective neuroprotective agent (memantine) and hippocampus-sparing WBRT technique [36, 37]. Chung et al. [15]. applied a lower WBRT dose with a boost to the tumour bed after metastasectomy, since WBRT neurotoxicity is associated with the total dose and fractionation [38]. Although no randomized studies have directly compared lower WBRT doses to the traditional WBRT dose of $30 \mathrm{~Gy}$, the advantage of lower WBRT dose has been shown in previous studies on prophylactic cranial irradiation (PCI). For example, a French study compared PCI (24 Gy in 8 fractions, EQD2 26 Gy) vs. no PCI, and observed no significant 
difference between the groups in terms of neuropsychological function or abnormalities [39]. In another trial performed at MD Anderson Cancer Centre, 30 patients received PCI (25 Gy in 10 fractions, EQD2 $26.04 \mathrm{~Gy}$ ) and no significant deterioration was observed after PCI [40]. Other approach to reduce the decrease of memory function could be the use of lower fraction doses.

Our aim was to find a balance in improving the survival with dose escalation to the macroscopic metastases, maintaining the intracranial control and reducing the probability of treatment-related cognitive decline; meanwhile keeping the treatment duration reasonable for patients even with multiple brain metastases. Therefore, we have applied conventional fractionation for $3 \mathrm{D}$ conformal whole brain and consecutive boost irradiation using the classical conventional fractionation scheme, $18 \times 2$ Gy +10 to $12 \times 2$ Gy up to $56-60$ Gy total dose for patients with relatively longer life expectancy. Later we have introduced a shortened regime of $15 \times 2.2$ Gy whole brain irradiation and simultaneously 0.7 Gy was delivered to the tumour or tumour bed after surgery. This technique allowed encompassing even 10-12 metastases into the boost volume, and lasted only 3 weeks, considered reasonable for patients with poorer condition. Tiwari and co-workers reported the results of SIB of 19 patients versus WBRT alone (13 patients), demonstrating better local control and improved survival in the SIB group [41]. Another publication from Italian groups emphasises the higher efficacy of the intensified treatment (WBRT and SRS boost), as well, enrolling 134 patients, of whom 21 were treated with SIB [28]. On the basis of high patient number, we could demonstrate the feasibility and clinical usefulness of dose escalation and in particular, the application of SIB in the management of BM, even for patients with unfavourable status. Direct comparison of SRS to SIB was performed in a matched cohort of 178 patients with similar baseline characteristics [42]. However, as for the OS, neither the treatment with SRS nor with SIB did result in any statistically significant difference, the SIB was associated with reduced intracranial impairment, likely due to the WBRT component of the treatment (HR $0.36, p<0.001$ ). Similarly for single and oligometastatic brain spread, several randomised clinical studies have proven the better outcome in terms of local and intracranial disease control with combined treatment approaches, such as surgery +WBRT, SRS + WBRT, or SIB; but in the majority of the cases these encouraging results could not be transformed into significantly improved survival [13, 15, 28, 42-44].

In contrast, our retrospective analysis confirmed the significant survival benefit for the whole group of patients including multiple metastases from intensified treatments without difference between the long and the shortened (SIB) regimes. This relevant survival difference was achieved not only for oligometastatic diseases, but for patients with multiple metastases ( $>4)$, as well. Hence, in the group that received SIB, one fifth of the patients had multiple metastases, in which the simultaneous boost was technically performable. The majority of evidence-based data derived from randomised clinical studies concerns patient population with single or oligometastases only, without progressing extracranial disease and in good status. Nevertheless, patients with worse initial parameters could not be enrolled - by forming homogeneous groups- into randomised trials. In fact, the design of clinical investigation on the management of brain metastasis is a highly challenging task. The application of the suggested recommendations of the Response Assessment in Neuro-Oncology (RANO) brain metastases group [45, 46] supposes patients in good condition and good cooperation, what is frequently not the case with patients of progressing extracranial cancer and/or low performance status. In our current study population, around half of the patients in the SIB arm had progressive disease and bad performance status $(<70 \%)$, according to Karnofsky score.

Our study has evident limitations. Its retrospective nature and consequent patient heterogeneity may have biased the results. Our effort to compare the outcome of the different treatment schemes by retrospectively dividing the patient population amongst similar prognostic groups could not completely compensate the lack of prospective patient enrolment. Furthermore, no objective assessment of late neurotoxicity has been performed. However, this study has several strengths. The large number of the patients allowed relevant statistical evaluation, and the three treatment approaches were clearly defined. Our aim to study the feasibility of SIB in 15 fractions even for patients, who cannot be enrolled into prospective clinical trials due to their bad prognostics, could be investigated. Conclusion could be drawn from this analysis on the applicability of lower WBRT fraction dose approach with a boost RT.

Therefore, considering all the limitations, our study on large patient series in RPA2 and RPA3 categories seems to document survival advantage of intensified irradiation schemes, which has high importance for the daily clinical decisions, even for patients in poor condition (KPS $<70 \%$ ). The novel generation of linear accelerators allow the introduction of fractionated stereotactic irradiation of the boost volumes simultaneously to the low fraction size $(<2.5 \mathrm{~Gy})$ WBRT.

\section{Conclusion}

The improvement in the systemic treatment of disseminated malignancies urges the establishment of optimal management of patients with brain metastasis with different clinical and tumour parameters. Meanwhile, some open questions could be hopefully answered soon by the results of the on-going trials on radiation technique and on combination of radiotherapy with targeted agents for patients with low number of BMs. 
Still, novel approaches are highly required to decrease the potential neurocognitive decline as a consequence of WBRT, since the majority of the patients belong to less favourable prognostic groups (i.e. with progressive extracranial status and multiple BMs). Therefore, retrospective analyses could provide valuable conclusions, even though they should be assessed critically. From our large series of evaluation, the intensive radiation approach for groups of BM patients seems to yield clinical benefit; and the feasibility of SIB in the management of BMs could be confirmed. Together with the use of neuroprotective agents and hippocampal-avoidance WBRT technique, smaller fraction size of WBRT simultaneously to fSRT boost is to be considered for further clinical investigation.

Contributions The contributions of the authors to the present study are as follows: (1) the conception and design of the study (Á.D., K.H.), (2) examination, therapy plans and follow-up of the patients (Á.D., Zs.E., A.M., K.H.), (4) radiological assessments (A.Cs.), (5) histopathological diagnostics (L.T., Z.R.), (6) neurosurgical treatment (P.B.), (7) radiotherapy planning physicist (E.F.), (8) collection and acquisition of data (Á.D.), (9) statistical analysis of data (Z.V.), (10) interpretation of data (Á.D., K.H.), (11) drafting the article (Á.D., K.H), (12) revising it critically for important intellectual content (K.H.).

All authors have approved the final version of the submission.

Funding This project was supported by Analytic Healthcare Quality User Information Programme of the National Research, Development and Innovation Fund, Hungarian Government, Grant No. VKSZ 12-12013-0012.

\section{Compliance with Ethical Standards}

Ethical Approval For this type of study (i.e. retrospective) formal consent is not required.

Informed Consent Informed consent was obtained from all individual participants included in the study.

Conflict of Interest The authors declare that they have no conflict of interest.

\section{References}

1. Nayak L, Lee EQ, Wen PY (2012) Epidemiology of brain metastases. Curr Oncol Rep 14(1):48-54

2. Singh M, Manoranjan B, Mahendram S, McFarlane N, Venugopal C, Singh SK (2014) Brain metastasis-initiating cells: survival of the fittest. Int J Mol Sci 15(5):9117-9133

3. Taillibert S, Le Rhun É (2015) Epidemiology of brain metastases. Cancer Radiother 19(1):3-9 (Article in French)

4. Soffietti R, Rudā R, Mutani R (2002) Management of brain metastases. J Neurol 249(10):1357-1369

5. Ellis TL, Neal MT, Chan MD (2012) The role of surgery, radiosurgery and whole brain radiation therapy in the management of patients with metastatic brain tumors. Int J Surg Oncol 952345

6. Khuntia D, Brown P, Li J et al (2006) Whole-brain radiotherapy in the management of brain metastasis. J Clin Oncol 24:1295-1304
7. Patchell RA, Tibbs PA, Walsh JW et al (1990) A randomized trial of surgery in the treatment of single metastases to the brain. N Engl J Med 322:494-500

8. Weber DC, Caparrotti F, Laouiti M, Malek K (2011) Simultaneous in-field boost for patients with 1 to 4 brain metastasis/es treated with volumetric modulated arc therapy: a prospective study on qualityof-life. Radiat Oncol 6:79

9. Linskey ME, Andrews DW, Asher AL et al (2010) The role of stereotactic radiosurgery in the management of patients with newly diagnosed brain metastases: a systematic review and evidencebased clinical practice guideline. J Neuro-Oncol 96(1):45-68

10. Gaspar LE, Mehta MP, Patchell RA et al (2010) The role of whole brain radiation therapy in the management of newly diagnosed brain metastases: a systematic review and evidencebased clinical practice guideline. J Neuro-Oncol 96(1):17-32

11. Kalkanis SN, Kondziolka D, Gaspar LE et al (2010) The role of surgical resection in the management of newly diagnosed brain metastases: a systematic review and evidencebased clinical practice guideline. J Neuro-Oncol 96(1):33-43

12. Sahgal A, Aayama H, Kocher M et al (2015) Phase 3 trials of stereotactic radiosurgery with or without whole-brain radiation therapy for 1 to 4 brain metastasis: individual patient data meta-analysis. Int J Radiat Oncol Biol Phys 91(4):710-717

13. Aayoma H, Tago M, Shirato H (2015) Stereotactic radiosurgery with or without upfront whole brain radiation therapy for one to four brain metastases. Secondary analysis of the diagnosis-specific graded prognostic assessment. JAMA Oncol 1(4):457-464

14. Yuan X, Liu WJ, Li B, Shen ZT, Shen JS, Zhu XX (2017) A Bayesian network meta-analysis of whole brain radiotherapy and stereotactic radiotherapy for brain metastasis. Medicine (Baltimore) 96(34):e7698. https://doi.org/10.1097/MD.0000000000007698

15. Chung SY, Chang JH, Kim HR, Cho BC, Lee CG, Suh CO (2017) Optimal dose and volume for postoperative radiotherapy in brain oligometastases from lung cancer: a retrospective study. Radiat Oncol J 35(2):153-162

16. Casanova N, Mazouni Z, Bieri S, Combescure C, Pica A, Weber DC (2010) Whole brain radiotherapy with a conformational external beam radiation boost for lung cancer patients with 1-3 brain metastasis: a multi institutional study. Radiat Oncol 5:13

17. Bruzzaniti V, Abate A, Pedrini M, Benassi M, Strigari L (2011) IsoBED: a tool for automatic calculation of biologically equivalent fractionation schedules in radiotherapy using IMRT with a simultaneous integrated boost (SIB) technique. J Exp Clin Cancer Res 30: 52

18. Borghetti P, Pedretti S, Spiazzi L, Avitabile R, Urpis M, Foscarini F, Tesini G, Trevisan F, Ghirardelli P, Pandini SA, Triggiani L, Magrini SM, Buglione M (2016) Whole brain radiotherapy with adjuvant or concomitant boost in brain metastasis:dosimetric comparison between helical and volumetric IMRT technique. Radiat Oncol 11:59. https://doi.org/10.1186/s13014-016-0634-6

19. Hall EJ, Brenner DJ (1993) The radiobiology of radiosurgery: rationale for different treatment regimes for AVMs and malignancies. Int J Radiat Oncol Biol Phys 25:381-385

20. Chao JH, Phillips R, Nickson JJ (1954) Roentgen-ray therapy of cerebral metastases. Cancer 7:682-689

21. Nieder C, Spanne O, Mehta MP, Grosu AL, Geinitz H (2011) Presentation, patterns of care, and survival in patients with brain metastases: what has changed in the last 20 years? Cancer 117(11): 2505-2512

22. Kocher M, Soffietti R, Abacioglu U et al (2011) Adjuvant wholebrain radiotherapy versus observation after radiosurgery or surgical resection of one to three cerebral metastases: results of the EORTC 22952-26001 study. J Clin Oncol 29(2):134-141

23. Tsao MN (2015) Brain metastases: advances over the decades. Ann Palliat Med 4(4):225-232 
24. Andrews DW, Scott CB, Sperduto PW et al (2004) Whole brain radiation therapy with or without stereotactic radiosurgery boost for patients with one to three brain metastases: phase III results of the RTOG 9508 randomised trial. Lancet 363:1665-1672

25. Kondziolka D, Patel A, Lunsford LD, Kassam A, Flickinger JC (1999) Stereotactic radiosurgery plus whole brain radiotherapy versus radiotherapy alone for patients with multiple brain metastases. Int J Radiat Oncol Biol Phys 45:427-434

26. Aoyama H, Shirato H, Tago M et al (2006) Stereotactic radiosurgery plus whole-brain radiation therapy vs stereotactic radiosurgery alone for treatment of brain metastases: a randomized controlled trial. JAMA 295:2483-2491

27. Kocher M, Soffietti R, Abacioglu U et al (2011) Adjuvant wholebrain radiotherapy versus observation after radiosurgery or surgical resection of one to three cerebral metastases: results of the EORTC 22952-26001 study. J Clin Oncol 29:134-141

28. Buglione M, Pedretti S, Gipponi S et al (2015) The treatment of patients with 1-3 brain metastases: is there a place for whole brain radiotherapy alone, yet? A retrospective analysis. Radiol Med 120(12):1146-1152

29. Brown PD, Asher AL, Ballman KV et al (2015) NCCTG N0574 (Alliance): A phase III randomized trial of whole brain radiation therapy (WBRT) in addition to radiosurgery (SRS) in patients with 1 to 3 brain metastases. J Clin Oncol 33(15 suppl):LBA4

30. Meyers CA, Smith JA, Bezjak A et al (2004) Neurocognitive function and progression in patients with brain metastases treated with whole-brain radiation and motexafin gadolinium: results of a randomized phase III trial. J Clin Oncol 22:157-165

31. Weiss SE, Kelly PJ (2010) Neurocognitive function after WBRT plus SRS or SRS alone. Lancet Oncol 11:220-221

32. Soffietti R, Kocher M, Abacioglu UM et al (2013) A European Organisation for Research and Treatment of Cancer phase III trial of adjuvant whole-brain radiotherapy versus observation in patients with one to three brain metastases from solid tumors after surgical resection or radiosurgery: quality-of-life results. J Clin Oncol 31: 65-72

33. Tsao M, Xu W, Sahgal A (2012) A meta-analysis evaluating stereotactic radiosurgery, whole-brain radiotherapy, or both for patients presenting with a limited number of brain metastases. Cancer 118:2486-2493

34. Soliman H, Das S, Larson DA, Sahgal A (2016) Stereotactic radiosurgery (SRS) in the modern management of patients with brain metastases. Oncotarget 7(11):12318-12330
35. Mehta M (2015) The controversy surrounding the use of wholebrain radiotherapy in brain metastases patients. Neuro-Oncology 17(7):919-923

36. Dye NB, Gondi V, Mehta MP (2015) Strategies for preservation of memory function in patients with brain metastases. Chin Clin Oncol 4(2):24

37. Brown PD, Pugh S, Laack NN et al (2013) Memantine for the prevention of cognitive dysfunction in patients receiving wholebrain radiotherapy: a randomized, double-blind, placebocontrolled trial. Neuro-Oncology 15(10):1429-1437

38. Filley CM, Kleinschmidt-DeMasters BK (2001) Toxic leukoencephalopathy. N Engl J Med 345:425-432

39. Arriagada R, Le Chevalier T, Borie F et al (1995) Prophylactic cranial irradiation for patients with small-cell lung cancer in complete remission. J Natl Cancer Inst 87:183-190

40. Komaki R, Meyers CA, Shin DM et al (1995) Evaluation of cognitive function in patients with limited small cell lung cancer prior to and shortly following prophylactic cranial irradiation. Int J Radiat Oncol Biol Phys 33:179-182

41. Tiwari V, Pande SC, Verma K, Goel S (2015) Simultaneous integrated boost with intensity modulated radiation therapy in brain oligometastases: a feasible technique for developing countries. South Asian J Cancer 4(1):11-14

42. Rodrigues G, Zindler J, Warner A, Bauman G, Senan S, Lagerwaard F (2013) Propensity-score matched pair comparison of whole brain with simultaneous in-field boost radiotherapy and stereotactic radiosurgery. Radiother Oncol 106(2):206-209

43. Patchell RA, Tibbs PA, Regine WF et al (1998) Postoperative radiotherapy in the treatment of single metastases to the brain: a randomized trial. JAMA 280(17):1485-1489

44. Wang TJ, Saad S, Qureshi YH et al (2015) Outcomes of gamma knife radiosurgery, bi-modality \&amp; tri-modality treatment regimens for patients with one or multiple brain metastases: the Columbia University Medical Center experience. J Neuro-Oncol 122(2):399-408

45. Lin NU, Lee EQ, Aoyama $\mathrm{H}$ et al (2013) Challenges relating to solid tumour brain metastases in clinical trials, part 1: patient population, response, and progression. A report from the RANO group. Lancet Oncol 14:e396-e406

46. Lin NU, Wefel JS, Lee EQ et al (2013) Challenges relating to solid tumour brain metastases in clinical trials, part 2: neurocognitive, neurological, and quality-of-life outcomes. A report from the RANO group. Lancet Oncol 14:e407-e416 